\title{
Low-frequency phonons in carbon nanotubes: A continuum approach
}

\author{
L. Chico, ${ }^{1}$ R. Pérez-Álvarez, ${ }^{2}$ and C. Cabrillo ${ }^{3}$ \\ ${ }^{1}$ Departamento de Física Aplicada, Facultad de Ciencias del Medio Ambiente, Universidad de Castilla-La Mancha, 45071 Toledo, Spain \\ ${ }^{2}$ Departamento de Física Teórica, Facultad de Física, Universidad de La Habana, 10400 Ciudad Habana, Cuba \\ ${ }^{3}$ Instituto de Estructura de la Materia, Consejo Superior de Investigaciones Científicas, C/ Serrano 123, 28006 Madrid, Spain
}

(Received 17 October 2005; revised manuscript received 30 December 2005; published 17 February 2006)

\begin{abstract}
Low-frequency phonons in carbon nanotubes are studied using a continuum model which allows consideration of an arbitrary wall thickness for the nanotube. Phonon dispersion relations are calculated for two archetypal examples of carbon nanotubes, the $(5,5)$ and $(10,10)$ tubes. The dependence of the radial breathing mode frequency at $\Gamma$ on the inverse nanotube diameter is verified within this model; furthermore, we prove it to hold for all pure modes within the thin-shell approximation. The effect of the nanotube wall thickness on the eigenfrequencies of carbon nanotubes is also analyzed, and a criterion to fix this parameter within a continuum model is presented. We compare our results to other continuum approaches, and show that by choosing the appropriate parameters, excellent agreement with recent first-principles calculations can be achieved.
\end{abstract}

DOI: 10.1103/PhysRevB.73.075425

PACS number(s): 68.65. $-\mathrm{k}, 63.22 .+\mathrm{m}$

\section{INTRODUCTION}

Vibrational properties of carbon nanotubes are the focus of increasing attention for various reasons. Besides the basic interest in the phonons of such intriguing quasi-onedimensional systems, ${ }^{1}$ detailed knowledge of the phonon spectrum of carbon nanotubes is a key ingredient for temperature-dependent transport, superconductivity, and optical characterization. Indeed, transport measurements at high electric fields indicated current saturation with increasing electric field, which can be understood in terms of electron-phonon interaction. ${ }^{2,3}$ Superconductivity in carbon nanotubes, reported in single-wall samples embedded in a zeolite matrix ${ }^{4}$ and in carbon nanotube ropes, ${ }^{5}$ has been explained within a conventional BCS theory of electronphonon coupling. But the main practical aspect for which vibrational modes of carbon nanotubes should be taken into account is their optical characterization: Raman spectra give vibrational information limited to the center of the Brillouin zone which can be used to discriminate among nanotubes of different diameters through the radial breathing modes (RBMs). ${ }^{6,7}$ Recently, a different way to assign chiral indices to carbon nanotubes has been developed by studying the resonant Raman intensity and frequency of radial breathing modes. ${ }^{8}$

Even though phonon dispersion relations for carbon nanotubes have been obtained using realistic models, ${ }^{9-12}$ the use of simpler approaches has proved to be rather fruitful: for example, Jishi et al. ${ }^{13}$ derived a widely used relation between the RBM frequency and the inverse tube diameter using a zone-folding method, and Popov et al. obtained the phonon spectrum of a $(10,10)$ nanotube using a force-constant model. ${ }^{14}$ Mahan and Jeon have also studied the vibrational modes of zigzag and armchair carbon nanotubes with a spring-and-mass model, ${ }^{15}$ analyzing the appropriate symmetry rules which give the correct flexure modes at low frequencies.

Continuum models have also been employed to investigate the vibrational properties of low-dimensional systems such as carbon nanotubes and fullerenes. ${ }^{16}$ Such models are a basic element in the formulation of electron-phonon interaction theories and allow for a better understanding of the physical properties of these systems. The longitudinal optical (LO) modes of infinite carbon nanotubes have been studied within the continuum approximation, ${ }^{17}$ allowing for the derivation of the optical deformation potential. However, it is well known that pure transverse or longitudinal modes are obtained in bulk homogeneous media; notwithstanding, in an interface problem or when boundary conditions in a finite region are imposed, modes have a mixed character. A continuum formulation which considered both longitudinal and transverse optical (TO) vibrations in a carbon nanotube was recently presented, ${ }^{18}$ demostrating that in general there is a considerable LO-TO mode mixing.

Low-frequency modes of infinite carbon nanotubes have also been studied within the continuum approximation: Suzuura and $\mathrm{Ando}^{19}$ derived a model for a cylindrical membrane, explicitly studying the effect of curvature, and formulated an electron-acoustic-phonon interaction Hamiltonian. Mahan ${ }^{20}$ obtained the classical vibrational modes of a thin hollow cylinder, thus considering the finite wall thickness of the nanotube. He solved the problem in the thin-wall limit; this allows one to obtain an analytical solution of the problem, but leaves aside the case of nanotubes with small diameter, for which the wall thickness cannot be considered small with respect to the nanotube radius. Very recently, Goupalov $^{21}$ applied group theory techniques to derive the equations of motion for the vibrations of two-dimensional graphite and carbon nanotubes in the thin-wall approximation. And in addition to carbon nanotubes, vibrational properties of infinite cylindrical hollow structures have also been studied employing continuum models; for example, mechanical properties and interface modes of microtubules of biological interest have been explored within a continuum approach. $^{22,23}$

Even though there is a considerable number of works that employ continuum models to describe the low-frequency modes of carbon nanotubes, to our knowledge all of them assume a small shell thickness. This approximation may not 
hold for small radii, and a discussion on the role of this parameter in continuum models is needed. In this work we obtain the low-frequency phonon dispersion relations of a hollow cylinder, particularizing for carbon nanotubes, without assuming beforehand any special value for the nanotube wall thickness. Results for the $(5,5)$ and $(10,10)$ tubes are presented and compared to results of other theoretical calculations. In order to improve quantitative agreement, we explored the dependence of the phonon spectrum on the nanotube thickness. We have found that it is an important parameter for some specific modes, which can be used as a guide to fix the shell width.

\section{MODEL}

The main assumption of a continuum model is that the properties of a low-dimensional structure can be described by bulk parameters. By applying appropriate boundary conditions to the bulk system, the modes of the low-dimensional system are obtained. Although the detailed atomic structure and geometry are lost, many basic features are correctly given. In fact, long-wavelength properties of bulk and lowdimensional systems are well described by a continuum approximation.

We assume that the bulk medium (graphene) is isotropic and homogeneous, and the basic parameters are the transverse and longitudinal velocities $v_{T}$ and $v_{L}$. With these parameters in mind, the equation of motion for the acoustic mechanical displacement $\vec{u}$ is given by ${ }^{24}$

$$
\omega^{2} \vec{u}=-v_{L}^{2} \vec{\nabla} \vec{\nabla} \cdot \vec{u}+v_{T}^{2} \vec{\nabla} \times \vec{\nabla} \times \vec{u} .
$$

This equation can be easily derived from the optical continuum model presented in Ref. 18 by a simple change of parameters; see Appendix A.

In a system with cylindrical symmetry modes can be labeled by a wave vector along the tube axis $k_{z}$, and an integer $n$ which gives the angular dependence of the modes and is related to the order $n$ of the Bessel functions appearing in the solutions. For the purpose of giving a compact expression for the eigenbasis it is useful to introduce the wave vectors

$$
\begin{gathered}
q_{T}^{2}=\frac{\omega^{2}}{v_{T}^{2}}-k_{z}^{2}, \\
q_{L}^{2}=\frac{\omega^{2}}{v_{L}^{2}}-k_{z}^{2} .
\end{gathered}
$$

A set of basis vectors for this problem can be found following the general method of potentials as shown in Refs. 25-27. For the sake of completeness, we give them explicitly here:

$$
\vec{u}_{L}=\left(\begin{array}{c}
f_{n}^{\prime}\left(q_{L} \rho\right) \\
\frac{i n}{q_{L} \rho} f_{n}\left(q_{L} \rho\right) \\
\frac{i k_{z}}{q_{L}} f_{n}\left(q_{L} \rho\right)
\end{array}\right) e^{i\left(n \varphi+k_{z} z\right)}
$$

$$
\begin{aligned}
& \vec{u}_{T 1}=\left(\begin{array}{c}
\frac{i k_{z}}{q_{T}} f_{n}^{\prime}\left(q_{T} \rho\right) \\
-\frac{n k_{z}}{q_{T}} \frac{1}{q_{T} \rho} f_{n}\left(q_{T} \rho\right) \\
f_{n}\left(q_{T} \rho\right)
\end{array}\right) e^{i\left(n \varphi+k_{z} z\right)}, \\
& \vec{u}_{T 2}=\left(\begin{array}{c}
i n \\
\frac{i n}{q_{T} \rho} f_{n}\left(q_{T} \rho\right) \\
-f_{n}^{\prime}\left(q_{T} \rho\right) \\
0
\end{array}\right) e^{i\left(n \varphi+k_{z} z\right)},
\end{aligned}
$$

where the vector components are understood in cylindrical coordinates $\left(u_{\rho}, u_{\varphi}, u_{z}\right)$; the prime denotes the derivative with respect to the argument and $f_{n}$ is an order- $n$ Bessel function of the first or second kind ( $J_{n}$ or $Y_{n}$, respectively), so for fixed $n$ and $k_{z}$ we have a six-function basis. $L$ and $T$ stand for longitudinal and transverse, respectively; ${ }^{28}$ indeed, it is straightforward to check that $\vec{\nabla} \times \vec{u}_{L}=0$ and $\vec{\nabla} \cdot \vec{u}_{T 1}=\vec{\nabla} \cdot \vec{u}_{T 2}=0$.

A general solution for the cylindrical hollow cylinder is a linear combination of the basis vectors which verifies the physical boundary condition of free surfaces, that is, the mechanical displacement at the inner and outer surfaces can be nonzero, but the momentum transmitted outside the cylinder should be zero. This condition can be cast in terms of the stress tensor: radial stress components at the inner and outer cylindrical surfaces must vanish. Detailed expressions of the stress tensor for an isotropic medium and the corresponding boundary conditions are given in Appendix B, from which the secular equation is obtained.

In any continuum model there is an infinite number of solutions which correspond to all possible indices $n$. When modeling a discrete system, the number of modes should be limited, keeping only a finite number. This cutoff is introduced by physical arguments: for acoustic phonons, direct comparison to discrete calculations in the frequency window of interest can set a natural cutoff for $n$.

There is an additional mode that verifies the equation of motion and the boundary conditions, but cannot be obtained by the above-mentioned method. It is a transverse mode with order $n=0$, for which the vibration is a pure twist around the tube circumference and it propagates with the bulk transverse velocity $v_{T}$ :

$$
\vec{u}_{T B} \propto\left(\begin{array}{l}
0 \\
\rho \\
0
\end{array}\right) e^{i k_{z} z}
$$

This mode was already described by Love, ${ }^{29}$ and has also been mentioned in more recent works on continuum models for carbon nanotube phonons. ${ }^{19-21}$ It has a linear dispersion relation $\omega=k v_{T}$ independent of the tube thickness and radius. Notice that this solution identically satisfies Neumann boundary conditions. 


\section{DESCRIPTION OF THE NANOTUBE ACOUSTIC MODES}

As the method of solution is formally equivalent to the one employed for optical phonons, ${ }^{18}$ the behavior of longitudinal and transverse solutions is obviously the same. We recall that it can be inferred from the structure of the basis vectors (4)-(6). We briefly summarize it here.

Axisymmetric (i.e., $n=0) L, T 1$, and $T 2$ modes are decoupled at $k_{z}=0$ : from Eqs. (4)-(6) it is easy to see that the vectors $\vec{u}_{L}, \vec{u}_{T 1}, \vec{u}_{T 2}$ with $k_{z}=0$ and $n=0$ have a single component in the $\vec{e}_{\rho}, \vec{e}_{z}, \vec{e}_{\varphi}$ directions, respectively. Thus, in this case the solutions are either pure radial breathing $(L)$, pure torsional (T2), or pure axial ( $T 1)$ modes. By "axial" mode we mean displacements along the cylinder axis, i.e., the $z$ direction. For $k_{z} \neq 0$, it can be seen from the basis vectors that $L-T 1$ mixing arises in axisymmetric modes. However, $T 2$ modes with $n=0$ retain their pure torsional character. This also applies for the bulk twisting mode $\vec{u}_{T B}$ above described and given in Eq. (7), which stays pure for $k_{z} \neq 0$.

As to flexural solutions $(n \neq 0), L-T 2$ coupling is expected even at $\Gamma$. However, for $k_{z}=0$, transverse $T 1$ modes are uncoupled from the rest and correspond to pure axial solutions. Finally, away from the center of the Brillouin zone, $L-T 1-T 2$ mixing is expected for flexural modes. To describe the precise dispersion relations in the general case, it is necessary to solve the problem numerically.

\section{LOW-FREQUENCY PHONONS IN CARBON NANOTUBES: NUMERICAL RESULTS}

To proceed with the numerical calculations we should choose the corresponding bulk parameters in the first place. The bulk material to which we are applying the hollow cylinder boundary conditions is graphene. The in-plane phonon dispersion relations for graphite are a good approximation for the graphene modes. From the experimental phonon dispersion relations ${ }^{30}$ we can extract the transverse and longitudinal sound velocities, $v_{T} \approx 14 \mathrm{~km} / \mathrm{s}$ and $v_{L} \approx 24 \mathrm{~km} / \mathrm{s}$. The fit was carried out in the region where the graphite LA and TA bands can be considered as linear, which is roughly for $k<1 \AA^{-1}$. This in turn sets the frequency cutoff around $400 \mathrm{~cm}^{-1}$ and, as we discussed in Sec. II, we should not consider frequencies far above this value.

As to the structural parameters, we model a nanotube as a hollow cylinder of inner radius $a=R-h$ and outer radius $b=R+h$. Having in mind the armchair $(N, N)$ nanotubes, we will take $R$ to be the calculated nanotube radius $R=3 N a_{\mathrm{C}-\mathrm{C}} /(2 \pi)$, where the carbon bond length is $a_{\mathrm{C}-\mathrm{C}}$ $=1.42 \AA$. The cylinder thickness $2 h$ is an important parameter of the model; in our previous work in optical phonons ${ }^{18}$ we took it to be equal to the graphite layer-to-layer distance $3.4 \AA$. Here we will initially take this value and then proceed to study the effect of nanotube wall thickness on the acoustic phonon spectrum.

We first discuss the results for a $(5,5)$ nanotube with $R=3.39 \AA$; in Fig. 1 the dispersion relations for this case are displayed. In the frequency window of interest we only find modes with $n=0,1,2$; there is no need to artificially select
$(5,5)$

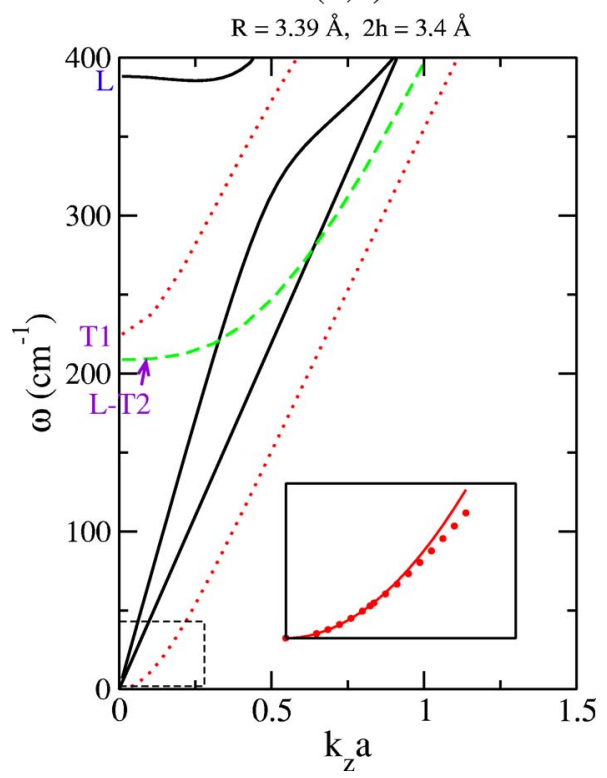

FIG. 1. (Color online) Acoustic phonon dispersion relations for a $(5,5)$ tube with $R=3.39 \AA, 2 h=3.4 \AA$. Axisymmetric bands are plotted with full (black) lines; bands with $n=1$ and 2 are plotted with dotted (red) and dashed (green) lines, respectively. The bands with nonzero frequency at $\Gamma$ are labeled according to their $L, T 1$, or $T 2$ character. Inset: Detail of the parabolic mode near $\Gamma$. The dots are the results of our calculation; the quadratic fit at low $k$ is depicted with a solid line.

the lowest- $n$ solutions. Let us examine the bands with zero frequency at $\Gamma$ : two of them have a linear dispersion, one is the twisting bulk mode with slope given by $v_{T}$ and the other one is a longitudinal acoustic band with slope close to $v_{L}$ when $\omega \rightarrow 0$; both are axisymmetric modes $(n=0)$. Both are also given by Goupalov ${ }^{21}$ and Suzuura and Ando; ${ }^{19}$ however, Mahan only predicts the twisting transverse mode. ${ }^{20}$

Particularly interesting is the acoustic mode with $n=1$. It appears to have a quadratic dispersion relation at small wave vectors. Such behavior was already discussed by Popov. ${ }^{14}$ Indeed, Mahan ${ }^{20}$ has demonstrated its $k^{2}$ dependence up to second order in the tube thickness $2 h$. Furthermore, Suzuura and Ando showed that without curvature effects, there are quadratic dispersion solutions for all nonzero $n$, but when curvature is taken into account they are shifted up at $\Gamma$ for all $n$ for except $n=1,{ }^{19}$ as we find here. Goupalov, ${ }^{21}$ however, has to introduce a corrective term in the equations of motion in order to get the right behavior for this mode, i.e., $\omega \rightarrow 0$ when $k_{z} \rightarrow 0$. The inset in Fig. 1 shows a quadratic fitting at low $k$ of our result. The excellence of the fit strongly supports a quadratic character independent of the thickness $2 h$, since in this case the thin-wall approximation is no longer valid $(2 h / R \approx 1$ for the case shown in Fig. 1). A quadratic dependence in the dispersion relation is not unique to nanotubes: it is also observed in graphite $^{31}$ in an out-of-plane acoustic mode of the single layer. In fact, this kind of dependence has also been described in classical elasticity theory; ${ }^{24}$ it arises in systems with at least one small dimension with respect to the wavelength. An interesting property of a onedimensional system with a quadratic acoustic dispersion is 


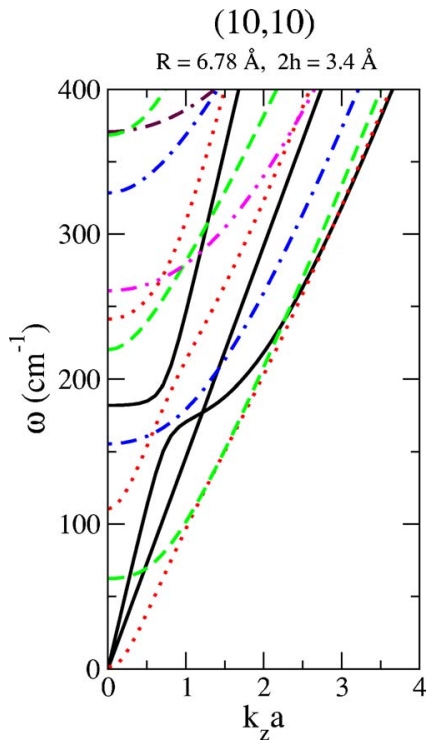

FIG. 2. (Color online) Acoustic phonon dispersion relations for a $(10,10)$ tube with $R=6.78 \AA, 2 h=3.4 \AA$. Bands with $n=0,1,2,3,4,5$ are plotted with solid (black), dotted (red), dashed (green), dot-dashed (blue), double-dot-dashed (magenta), and double-dash-dotted (maroon) lines, respectively.

that it yields a divergence in the density of phonon modes at $\omega=0$. This has dramatic thermodynamic consequences at low temperatures. ${ }^{32}$

Among the rest of the bands that have a nonzero frequency at $\Gamma$, the most relevant one is the radial breathing mode, with $n=0$, which for this narrow tube appears at rather high frequencies. The repulsion with the formerly described $n=0$ band with linear dispersion at the origin is quite evident, marking the deviation from linear behavior. Such repulsion does not exist between this band and the torsional bulk mode, even though they have the same order, i.e., angular momentum. We find the same behavior in the dispersion relations obtained by other authors. ${ }^{19,21}$ This result, at first sight surprising, can be understood by recalling that the twisting bulk mode (7) does not belong to the subspace of solutions spanned by the vectors (4)-(6). The only band with $n=2$ appearing in this frequency window has a frequency at $\Gamma$ around $205 \mathrm{~cm}^{-1}$. This is one of the parabolic bands described by Suzuura and Ando ${ }^{19}$ which are shifted to nonzero frequencies when curvature effects are considered. Thus we confirm the importance of curvature, especially for smallradius nanotubes.

Let us proceed with the results for the $(10,10)$ tube. This case is far more interesting because the majority of theoretical calculations choose this tube as an example, motivated by the great amount of experimental data attributed to this particular nanotube. Given that the diameter is doubled, we will consider higher-order solutions in the dispersion relations. In Fig. 2 the phonon band structure for a $(10,10)$ tube is presented; the radius is $R=6.78 \AA$ and the wall thickness is $2 h=3.4 \AA$. Doubling the radius has the expected effect of lowering the frequencies at $\Gamma$ of all the branches. This can be easily understood as the consequence of the smaller confinement in larger tubes. The overall picture agrees with other

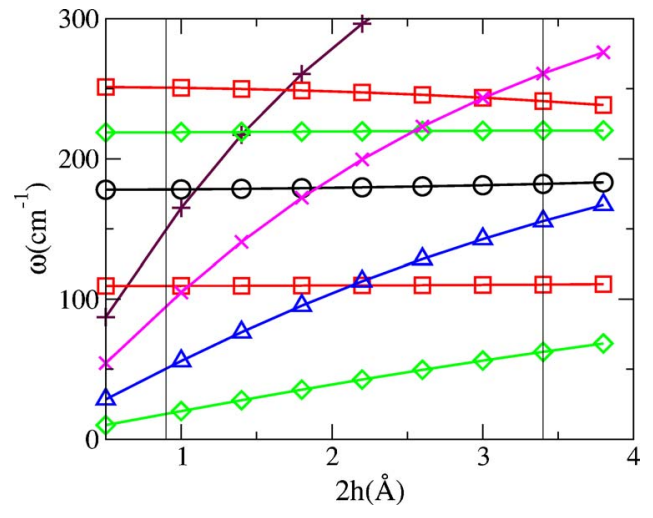

FIG. 3. (Color online) Variation of the phonon frequencies at $\Gamma$ with the nanotube wall thickness for a $(10,10)$ tube. As a guide to the eye, two thin full lines indicate the values corresponding to Fig. 2, i.e., $2 h=3.4 \AA$, as well as the fit $(2 h=0.9 \AA)$. The symbols (colors) indicate the order of the mode: $n=0$, circles (black); $n=1$, squares (red); $n=2$, diamonds (green); $n=3$, triangles (blue); $n=4$, crosses (magenta); $n=5$, plus signs (maroon).

theoretical results; let us see whether quantitative agreement can also be achieved. We see that the radial breathing mode frequency has a frequency of $182 \mathrm{~cm}^{-1}$, which is nicely close to the experimental result ${ }^{6}$ of $186 \mathrm{~cm}^{-1}$, and within the range of other theoretical calculations. . $^{70,12}$

The analysis of other bands indicates that some tuning is necessary. The lowest nonzero frequency at $\Gamma$ is too high in our results. Let us note, however, that the dispersion in the theoretical values for this mode is non-negligible: the lowfrequency dispersion relations given by $a b$ initio calculations can be affected by the interaction cutoff in the force-constant approach. The recent work by $\mathrm{Ye}$ and co-workers ${ }^{12}$ gives a rather high value, around $40 \mathrm{~cm}^{-1}$; but notice this $E_{2 g}$ mode was reported to be around $20 \mathrm{~cm}^{-1}$ by other authors. $14,19,33$ This latter value of the lowest nonzero frequency is further substantiated by molecular dynamics calculations ${ }^{34}$ of the density of modes, which yield a value of $17 \mathrm{~cm}^{-1}$.

To achieve quantitative agreement, especially for lowfrequency modes at $\Gamma$, we have studied the dependence on the tube wall thickness. In Fig. 3 we show the variation of the mode frequencies at the center of the Brillouin zone with respect to $2 h$ for a $(10,10)$ tube $(R=6.78 \AA)$. We find that several modes do not depend on the wall thickness; in particular this happens to the RBM. This can be understood by recalling the geometry of this vibration. Other modes do change noticeably with $2 h$, and even the ordering of the modes can be affected by this parameter. If we want to fit the $E_{2 g}$ frequency at $\Gamma$ around $17 \mathrm{~cm}^{-1}$, we have $2 h=0.9 \AA$. This thickness is closer to the $\sigma$ orbital size of carbon than to the van der Waals distance previously used.

Let us reexamine the phonon dispersion relations for the $(10,10)$ nanotube, but with the fitted wall thickness. In Fig. 4 we present our calculated results for the $(10,10)$ tube with $2 h=0.9 \AA$, along with the $a b$ initio calculations that we have digitalized from Ref. 12. We see that the agreement at $k_{z}$ $\approx 0$ is excellent, especially considering there was no fine tuning of the fitting parameter. A fine tuning - not only of $2 h$, but also of $v_{L}$ and $v_{T}$-would probably lead to an improved 


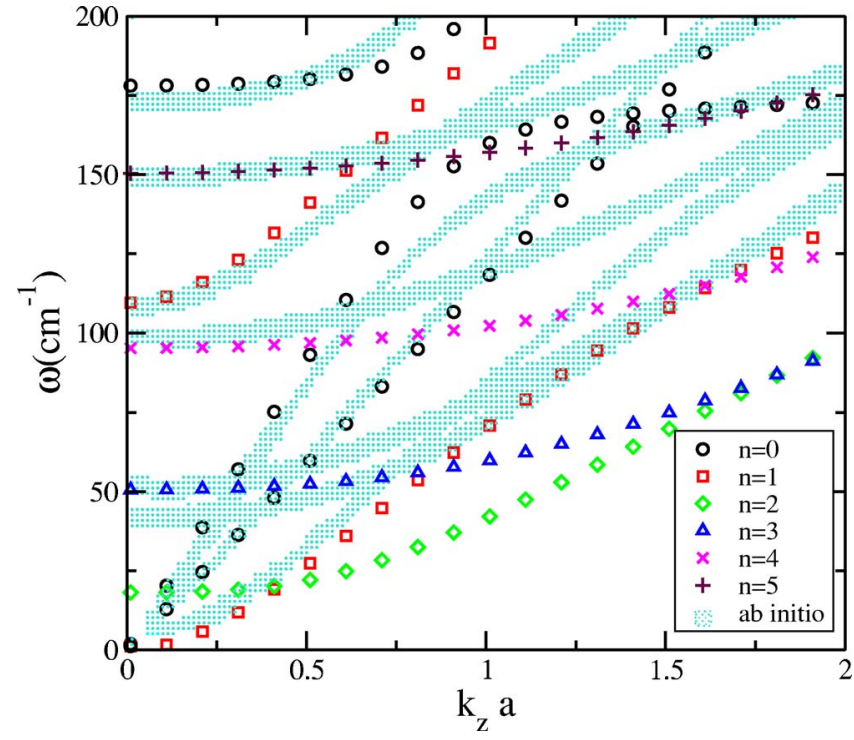

FIG. 4. (Color online) Acoustic phonon dispersion relations for a $(10,10)$ tube with $R=6.78 \AA$, and fitted wall thickness $2 h=0.9 \AA$ A. Our results are compared to the $a b$ initio calculation by Ye et al., digitalized from Ref. 12.

agreement. Obviously, there is a clear mismatch in the lowest finite-frequency mode, since our fitting criterion was independent of the result given by Ye et al. for this mode. But in fact we would have obtained the same fit if we had chosen the other $h$-dependent modes with $n=3,4,5$. The fact that the overall agreement is remarkably good supports the validity of both calculations, ours and that of Ref. 12. We consider that the discrepancy for the lowest nonzero mode might indicate that some problems still remain in the $a b$ initio approach for the low-frequency region. Notice also that Suzuura and Ando ${ }^{19}$ predict an order-5 mode almost degenerate with the RBM at $\Gamma$; this is not given by the ab initio results $^{12}$ nor by our model. This indicates that the finite wall thickness does have a role in a continuum approach.

We have also studied the frequency dependence on $h$ for other tubes. In Fig. 5 we present the frequency dependence on $2 h$ for two other cases, namely, the $(5,5)$ and the $(6,6)$ armchair tubes. Our results are shown together with the calculated values extracted from Ref. 12. We see that the value $2 h=0.9 \AA$ nicely fits the $a b$ initio results, so within our model one can expect that the tube thickness should be approximately the same for all tubes. As mentioned above, this value is close to the $\sigma$ orbital size in graphite. These are the orbitals responsible for in-plane bonding in graphene, and one should not expect this value to vary much when rolling the graphene layer into a tube. In this light, our results may indicate that the key factor as to elastic properties of nanotubes are the $\sigma$ in-plane bonds.

From these results, a criterion to fix the nanotube thickness in a continuum model can be inferred. The lowest nonzero-frequency modes with $n \geqslant 2$ have a significant dependence on $h$, and they can be used to fix this parameter. Within our continuum model it is preferable to use the $E_{2 g}$ mode or, if this value is not available or reliable, the abovementioned $n>2$ modes in the range $0-100 \mathrm{~cm}^{-1}$. The reason
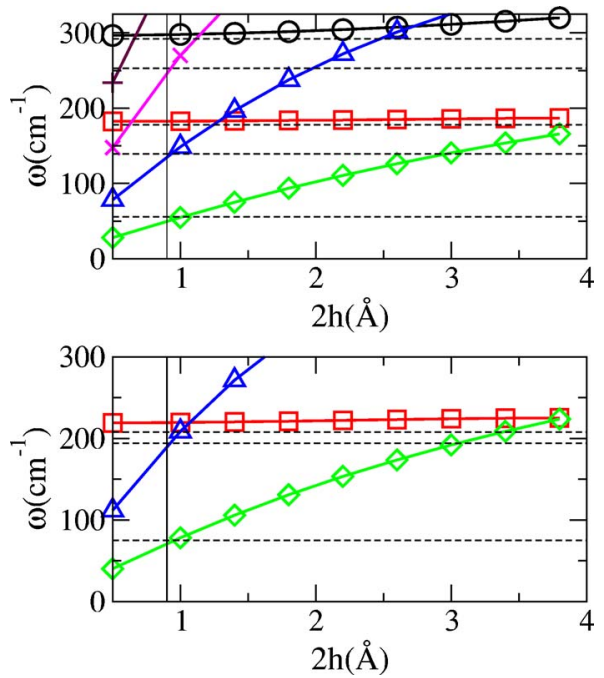

FIG. 5. (Color online) Variation of phonon frequencies at $\Gamma$ with nanotube thickness for the $(5,5)$ (bottom) and the $(6,6)$ (top) nanotubes. Symbols (colors) indicate the order of the mode: $n=0$, circles (black); $n=1$, squares (red); $n=2$, diamonds (green); $n=3$, triangles (blue). As a guide to the eye, a thin full line marks the thickness value $2 h=0.9 \AA$. Thin dashed lines show the frequencies as calculated by Ye et al. in Ref. 12 .

to prefer this low-frequency region is that the graphene bands entering the model were modeled as linear. Such approximation is excellent for the low-frequency spectrum, but deviations from parabolicity can be expected for higher frequencies.

Finally, we have verified that our continuum model does predict the linear relation between the RBM frequency and the inverse diameter of the nanotube. In fact, it predicts that this linear relationship holds for any pure mode at $\Gamma$. This can be easily understood by recalling some features of the secular equation. It depends on Bessel functions and its derivatives evaluated in any of the following arguments: $q_{L} a$, $q_{L} b, q_{T} a, q_{T} b$. For pure modes, the secular equation depends only on two arguments, either $q_{L} a, q_{L} b$ or $q_{T} a, q_{T} b$. Thus, such equations can be cast in terms of the arguments $q_{L}(d$ $-h / 2), q_{L}(d+h / 2)$ or $q_{T}(d-h / 2), q_{T}(d+h / 2)$, depending on the transverse or longitudinal character of the solution. For $h$ small, these transcendent equations are functions of either $q_{L} d$ or $q_{T} d$. Hence the behavior $\omega \approx C / d$ for pure acoustic modes. A similar argument can be applied to the optical phonon calculation, yielding a slightly different expression for pure optical modes.

In Fig. 6 we present the frequency at $\Gamma$ of several modes versus the tube radius in a log-log plot. Calculations were done for a fixed shell thickness of $3.4 \AA$.

We see that our continuum model does yield the $1 / d$ behavior for the RBM predicted by Jishi et al. ${ }^{13}$ and later verified by different theoretical methods, which constitutes one of the main features studied in the experimental characterization of carbon nanotubes. Concentrating on the higher-radii nanotubes $(N=10-15)$ for which the small $h$ approximation is better, we obtain 


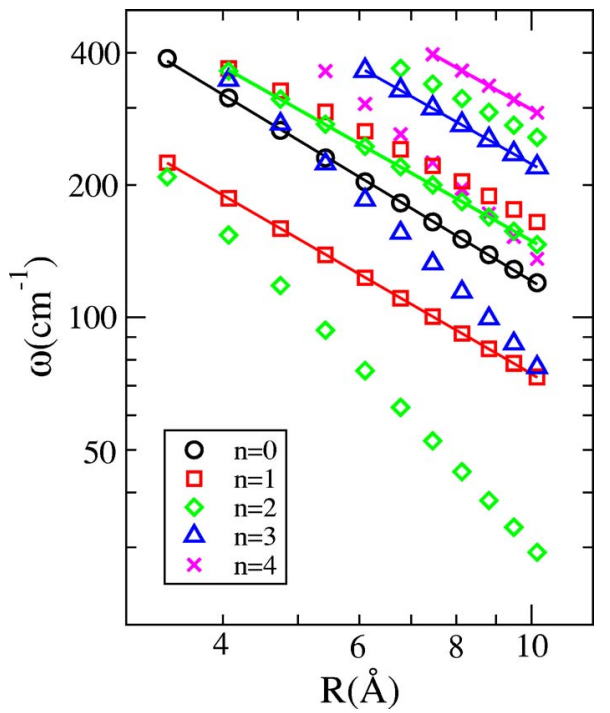

FIG. 6. (Color online) Log-log plot of the phonon frequencies at $\Gamma$ as a function of the radius for a series of $(N, N)$ nanotubes from $N=5$ to 15 . A fit to a $\approx 1 / d$ law is shown for those modes which approximately follow it.

$$
\omega_{R B M}=\frac{2460 \mathrm{~cm}^{-1} \AA}{d} .
$$

This value is within the range found by other methods. ${ }^{35}$ As discussed before, the 1/d law is verified not only by the RBM, but for several other modes. Our model predicts that only modes with a mixed character deviate from the $1 / d$ behavior. One example is the lowest-frequency mode with $n=2$, plotted with diamonds in Fig. 2. This behavior of the lowest $E_{2 g}$ Raman-active mode was also reported by other authors. ${ }^{33}$ With our model we can relate such behavior to its mixed $L-T$ character, and extending our analysis to all modes (that is, not only to those which are Raman active), we find several which do not follow the $1 / d$ law, as can be clearly seen in Fig. 5.

\section{CONCLUSIONS}

We have presented a continuum model for acoustic phonons in carbon nanotubes, valid for arbitrary shell thickness, which shows excellent agreement with experimental results and recent $a b$ initio calculations. We have studied the frequency dependence on the nanotube thickness, finding that several modes do have a strong dependence on this parameter. From these results, we give a criterion to fix the nanotube wall thickness in a continuum model. We have also verified the well-known relation between the RBM and the inverse diameter. Furthermore, we have shown that such behavior should be followed for all pure transverse or longitudinal modes at $\Gamma$, provided that the thin-shell approximation is valid. By introducing the nanotube wall thickness as another parameter without making any particular assumptions on its value, we have been able to improve the quantitative agreement given by continuum models and clarified its importance in the nanotube phonon spectrum.

\section{ACKNOWLEDGMENTS}

We thank Professor C. Trallero-Giner and Dr. Ricardo Fernández-Perea for helpful discussions. This work has been partly supported by the Spanish DGES (Grant No. MAT 2002-04540-C05-03) and the Vicerrectorado de Relaciones Internacionales (UCLM). L.C. acknowledges hospitality at the Universidad de La Habana and R.P.-A. acknowledges hospitality at the ICAM-UCLM.

\section{APPENDIX A: DERIVATION OF THE ACOUSTIC EQUATIONS OF MOTION FROM THE OPTICAL CONTINUUM MODEL}

Let us briefly recall the main ingredients of our model for optical phonons: we assumed that the bulk medium is isotropic and homogeneous, and its optical modes are given by parabolic dispersion relations. Thus, the basic parameters are the transverse and longitudinal bulk frequencies at the center of the Brillouin zone $(\Gamma), \omega_{T}$ and $\omega_{L}$, and the corresponding parabolicity parameters, $\beta_{T}$ and $\beta_{L}$, which can be fitted to experimental dispersion relations; for nonpolar media, $\omega_{L}=\omega_{T} \equiv \omega_{0}$. by $^{18}$

For optical phonons the equations of motion are given

$$
\left(\omega^{2}-\omega_{0}^{2}\right) \vec{u}=\beta_{L}^{2} \vec{\nabla} \vec{\nabla} \cdot \vec{u}-\beta_{T}^{2} \vec{\nabla} \times \vec{\nabla} \times \vec{u} .
$$

The equations for acoustic phonons (1) can be obtained from Eq. (A1) by a simple change of parameters: bulk frequencies at $\Gamma$ are set equal to zero $\left(\omega_{0}=0\right)$, and the squared values of the parabolicity parameters $\beta_{T}^{2}, \beta_{L}^{2}$ are chosen as negative values which model the linear dispersion relations of the bulk acoustic branches, i.e., $\beta_{T}^{2} \rightarrow-v_{T}^{2}, \beta_{L}^{2} \rightarrow-v_{L}^{2}$. This sets a natural frequency cutoff, namely, the frequency window for which such a linear approximation for bulk bands can be made. The appropriateness of our procedure is confirmed by the fact that the equations of motion (1) are equal to those derived from elasticity theory. ${ }^{29}$

\section{APPENDIX B: BOUNDARY CONDITIONS AND SECULAR EQUATION} as $^{36}$

The stress tensor $\overrightarrow{\vec{\sigma}}$ for an isotropic medium can be written

$$
\overrightarrow{\vec{\sigma}}=\rho_{m}\left(2 v_{T}^{2}-v_{L}^{2}\right) \vec{\nabla} \cdot \overrightarrow{\vec{u}} \overrightarrow{\vec{I}}-\rho_{m} 2 v_{T}^{2} \vec{\nabla} \vec{u}
$$

where $\rho_{m}$ is the mass density. The free surface boundary conditions imply that the radial stress components $\sigma_{\rho \rho}, \sigma_{\rho \varphi}$, and $\sigma_{\rho z}$ vanish at the inner and outer cylindrical surfaces. ${ }^{29}$ Denoting the inner radius and outer radii by $a$ and $b$ respectively, this gives

$$
\left(\left(2 v_{T}^{2}-v_{L}^{2}\right)(\vec{\nabla} \cdot \vec{u})-2 v_{T}^{2} \frac{\partial u_{\rho}}{\partial \rho}\right)_{\rho=a, b}=0
$$




$$
\begin{gathered}
{\left[\frac{1}{\rho} \frac{\partial u_{\rho}}{\partial \varphi}+\rho \frac{\partial}{\partial \rho}\left(\frac{u_{\varphi}}{\rho}\right)\right]_{\rho=a, b}=0} \\
\left(\frac{\partial u_{\rho}}{\partial z}+\frac{\partial u_{z}}{\partial \rho}\right)_{\rho=a, b}=0 .
\end{gathered}
$$

A general solution to the problem is a linear combination of the basis vectors (4)-(6):

$$
\vec{u}=\sum_{M} A_{M}^{+} \vec{u}_{M}^{+}+\sum_{M} A_{M}^{-} \vec{u}_{M}^{-}
$$

Here $M$ runs over the modes $L, T 1$ or $T 2$, and,+- indicates that the Bessel function $f_{n}$ is $J_{n}$ or $Y_{n}$, respectively. Imposing the boundary conditions (B2)-(B4) to this eigenfunction, we have a homogeneous linear system for the six coefficients. The eigenfrequencies are calculated by setting the corresponding secular determinant equal to zero: for each frequency, the eigenmodes are found up to normalization by solving for the six coefficients.
${ }^{1}$ R. Saito, G. Dresselhaus, and M. S. Dresselhaus, Physical Properties of Carbon Nanotubes (Imperial College Press, London, 1998).

${ }^{2}$ Z. Yao, C. L. Kane, and C. Dekker, Phys. Rev. Lett. 84, 2941 (2000).

${ }^{3}$ P. G. Collins, M. Hersam, M. Arnold, R. Martel, and Ph. Avouris, Phys. Rev. Lett. 86, 3128 (2001).

${ }^{4}$ Z. K. Tang, L. Zhang, N. Wang, X. X. Zhang, G. H. Wen, G. D. Li, J. N. Wang, C. T. Chan, and P. Sheng, Science 292, 2462 (2001).

${ }^{5}$ M. Kociak, A. Yu. Kasumov, S. Guéron, B. Reulet, I. I. Khodos, Yu. B. Gorbatov, V. T. Volkov, L. Vaccarini, and H. Bouchiat, Phys. Rev. Lett. 86, 2416 (2001).

${ }^{6}$ A. M. Rao, E. Richter, S. Bandow, B. Chase, P. C. Eklund, K. A. Williams, S. Fang, K. R. Subbaswamy, M. Menon, A. Thess, R. E. Smalley, G. Dresselhaus, and M. S. Dresselhaus, Science 275, 187 (1997).

${ }^{7}$ E. Richter and K. R. Subbaswamy, Phys. Rev. Lett. 79, 2738 (1997).

${ }^{8}$ H. Telg, J. Maultzsch, S. Reich, F. Hennrich, and C. Thomsen, Phys. Rev. Lett. 93, 177401 (2004).

${ }^{9}$ J. Yu, R. K. Kalia, and P. Vashishta, J. Chem. Phys. 103, 6697 (1995)

${ }^{10}$ D. Sánchez-Portal, E. Artacho, J. M. Soler, A. Rubio, and P. Ordejón, Phys. Rev. B 59, 12678 (1999).

${ }^{11}$ O. Dubay and G. Kresse, Phys. Rev. B 67, 035401 (2003).

${ }^{12}$ L.-H. Ye, B.-G. Liu, D.-S. Wang, and R. Han, Phys. Rev. B 69, 235409 (2004).

${ }^{13}$ R. A. Jishi, L. Venkataraman, M. S. Dresselhaus, and G. Dresselhaus, Chem. Phys. Lett. 209, 77 (1993).

${ }^{14}$ V. N. Popov, V. E. Van Doren, and M. Balkanski, Phys. Rev. B 61, 3078 (2000).

${ }^{15}$ G. D. Mahan and G. S. Jeon, Phys. Rev. B 70, 075405 (2004).

${ }^{16}$ M. A. Stroscio and M. Dutta, Phonons in Nanostructures (Cambridge University Press, Cambridge, U.K., 2001).

${ }^{17}$ M. Stroscio, M. Dutta, D. Kahn, and K. W. Kim, Superlattices Microstruct. 29, 405 (2001).

${ }^{18}$ L. Chico and R. Pérez-Álvarez, Phys. Rev. B 69, 035419 (2004).

${ }^{19}$ H. Suzuura and T. Ando, Phys. Rev. B 65, 235412 (2002).
${ }^{20}$ G. D. Mahan, Phys. Rev. B 65, 235402 (2002).

${ }^{21}$ S. V. Goupalov, Phys. Rev. B 71, 085420 (2005).

${ }^{22}$ Y. M. Sirenko, M. A. Stroscio, and K. W. Kim, Phys. Rev. E 53, 1003 (1996).

${ }^{23}$ Y. M. Sirenko, M. A. Stroscio, and K. W. Kim, Phys. Rev. E 54, 1816 (1996).

${ }^{24}$ L. D. Landau and E. M. Lifshitz, Theory of Elasticity, Course in Theoretical Physics Vol. 7 (Pergamon, Oxford, 1986).

${ }^{25}$ C. Trallero-Giner, R. Pérez-Álvarez, and F. García-Moliner, Long Wave Polar Modes in Semiconductor Heterostructures (Elsevier Science, Oxford, 1998).

${ }^{26}$ P. M. Morse and H. Feshbach, Methods of Theoretical Physics (McGraw-Hill, New York, 1953).

${ }^{27}$ E. Roca, C. Trallero-Giner, and M. Cardona, Phys. Rev. B 49, 13704 (1994).

${ }^{28}$ We emphasize that by longitudinal we mean irrotational $\left(\vec{\nabla} \times \overrightarrow{u_{L}}\right.$ $=0)$ and, consequently, a transverse field is solenoidal $(\vec{\nabla} \cdot \vec{u}$ $=0)$; see, for example, Ref. 24. Certainly, in isotropic infinite media a longitudinal wave does vary in the direction of propagation, and a transverse wave field oscillates perpendicularly to the direction of propagation. The boundary conditions for a cylindrical shell make the system anisotropic and this simple correspondence no longer holds. Thus, the radial breathing mode is longitudinal $\left(\vec{\nabla} \times \vec{u}_{R B M}=0\right)$, although it is an oscillation perpendicular to the direction of propagation.

${ }^{29}$ A. E. H. Love, A Treatise on the Mathematical Theory of Elasticity (Dover, New York, 1944), pp. 288-292.

${ }^{30}$ C. Oshima, T. Aizawa, R. Souda, Y. Ishizawa, and Y. Sumiyoshi, Solid State Commun. 65, 1601 (1988).

${ }^{31}$ R. Nicklow, N. Wakabayashi, and H. G. Smith, Phys. Rev. B 5, 4951 (1972).

${ }^{32}$ V. N. Popov, Phys. Rev. B 66, 153408 (2002).

${ }^{33}$ R. Saito, T. Takeya, T. Kimura, G. Dresselhaus, and M. S. Dresselhaus, Phys. Rev. B 57, 4145 (1998).

${ }^{34} \mathrm{R}$. Fernández-Perea (private communication)

${ }^{35} \mathrm{~S}$. Reich, C. Thomsen, and J. Maultzsch, Carbon Nanotubes (Wiley-VCH, Berlin, 2004), p. 142.

${ }^{36}$ F. Comas and C. Trallero-Giner, Physica B 192, 394 (1993). 